Document downloaded from:

http://hdl.handle.net/10251/154803

This paper must be cited as:

Puche Panadero, M.; Velty, A. (2019). Readily available Ti-beta as an efficient catalyst for greener and sustainable production of campholenic aldehyde. Catalysis Science \& Technology. 9(16):4293-4303. https://doi.org/10.1039/c9cy00957d

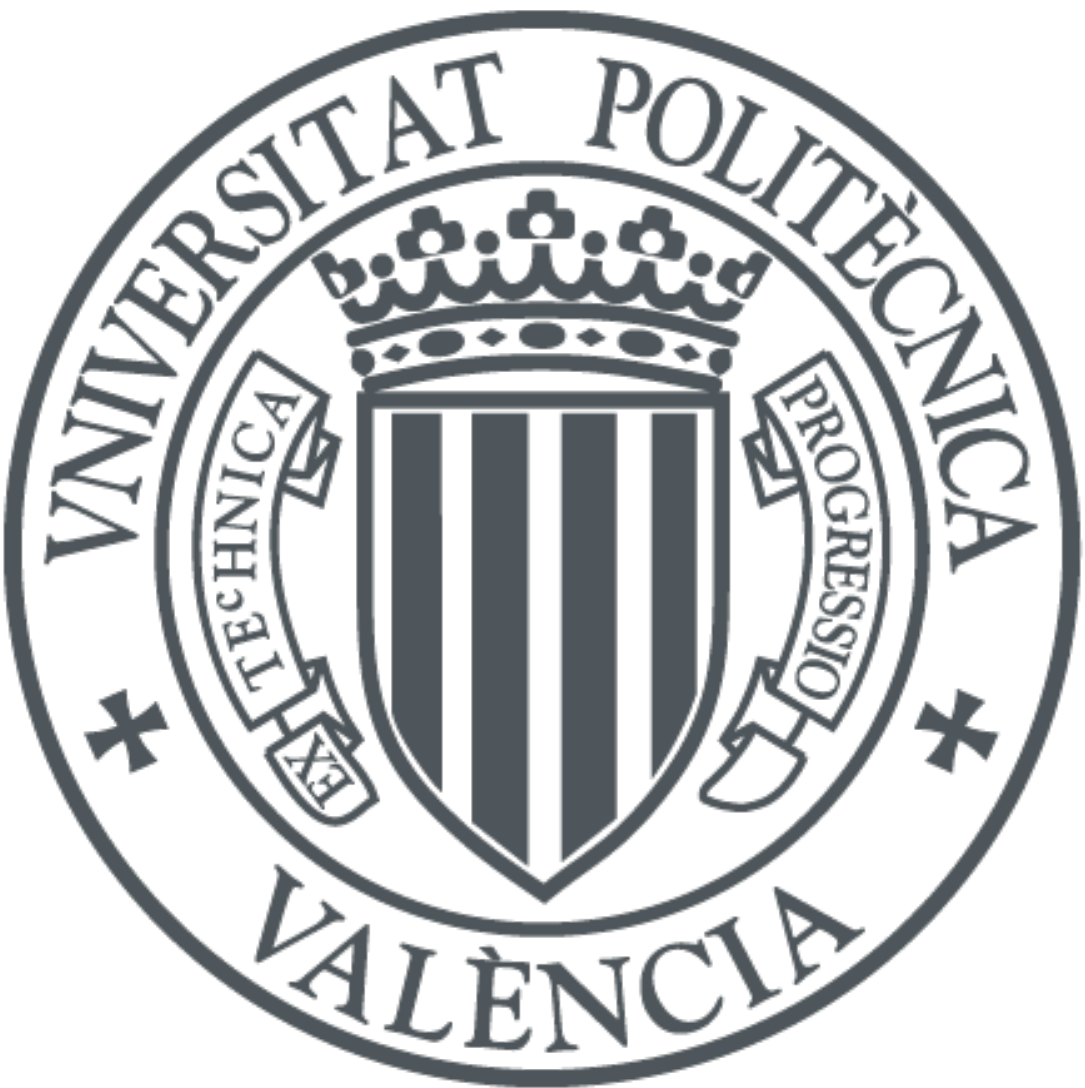

The final publication is available at

https://doi.org/10.1039/c9cy00957d

Copyright The Royal Society of Chemistry

Additional Information 


\section{Readily available Ti-Beta as efficient catalyst for greener and sustainable production of campholenic aldehyde}

Received 00th January 20xx, DOI: $10.1039 / \times 0 \times x 00000 x$ Accepted 00th January 20xx

\begin{abstract}
Marta Puche Panadero and Alexandra Velty*
Different Ti-Beta zeolite samples were prepared following a convenient and optimized post-synthetic route and starting from commercial Al-Beta zeolite. Lewis acid sites has been successfully incorporated into the vacant tetrahedral (T)-sites of a dealuminated Beta-framework by ball-milling solid-state ion-exchange. A tribology-ball milling process was used in order to increase the interaction between dealuminated-Beta zeolite and Ti-precursor. Thermal treatments with water and aqueous solution of $\mathrm{NaNO}_{3}$ or $\mathrm{Li} \mathrm{NO}_{3}$ allowed to optimize the catalytic properties of the Ti- Lewis active sites which exhibited excellent catalytic activity and stability for the isomerization of $\alpha$-pinene oxide into campholenic aldehyde in both batch and fixed bed reactor systems. Additionally, the catalytic performance of post-synthesised Ti-Beta was compared to a Ti-beta prepared in fluoride media. From different point of views such as the preparation of readily, highly active, selective and stable catalyst, throughput, sustainability and cost, herein we report the selective solid catalysed $\alpha$-PO isomerization with excellent results, $88 \%$ selectivity and yield, CA production of $225 \mathrm{~g} / \mathrm{g}_{\text {cat }} / \mathrm{h}$ and new opportunities.
\end{abstract}

\section{Introduction}

In response to the needs of sustainable chemistry and the use of renewable feedstock, terpenes offer a large and versatile class of organic compound that are widely found in essential oils and are useful for fine chemicals industry. One of the most important source of terpenes is the turpentine oleoresin, extracted from coniferous trees whose main components are $\alpha$ - and $\beta$-pinene. Campholenic aldehyde (CA) is an important intermediate in the synthesis of

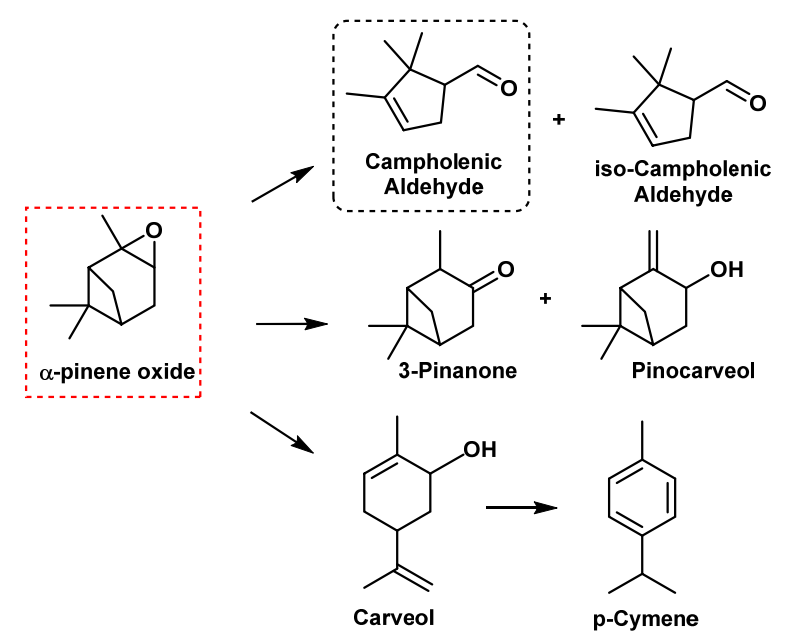

Scheme 1. Main products of isomerization of $\alpha$-pinene oxide.

Instituto de Tecnología Química, Universitat Politècnica de València-Consejo Superior de Investigaciones Científicas, Avenida de los Naranjos s/n, E-46022 Valencia, Spain. E-mail: avelty@itq.upv.es, Tel: +34963879697.

+ Footnotes relating to the title and/or authors should appear here.

Electronic Supplementary Information (ESI) available: [details of any supplementary information available should be included here]. See DOI: 10.1039/x0xx00000x several sandalwood fragrances and can be obtained through twosteps synthesis from $\alpha$-pinene. In the first step the epoxide is obtained, being the $\alpha$-pinene oxide isomerized, preferably in the presence of an acid catalyst, in a second step, providing different products of high-added value (Scheme 1). The isomerization has been widely studied in the last years in the presence of homogeneous and heterogeneous acid catalysts providing different products through competitive and consecutive reactions [1-3]. Several studies reported that Lewis acids favour the formation of campholenic, iso-campholenic aldehydes and trans-3-pinanone, while Brønsted acids result in the carveols and p-cymene. Selective preparation of campholenic aldehyde is conventionally catalysed by homogeneous Lewis acids such as $\mathrm{ZnCl}_{2}$ and $\mathrm{ZnBr}_{2}$ giving up to $85 \%$ yield of the desired aldehyde. However, there are numerous associated drawbacks such as a rapid deactivation of the catalysts or the production of large amount of contaminated waste with heavy metal due to the neutralisation step and the no opportunity of recycling [4-6]. Being numerous the advantages of the heterogeneous catalysts, different studies using solid Lewis acids have been reported. Likewise, Van Bekkum and co-workers reported the high catalytic performance of free Al Ti-beta zeolite to perform the isomerization of $\alpha$-pinene oxide, with CA selectivity up to $89 \%$ in the liquid phase and up to $94 \%$ in the gas phase. Indeed, among nanoporous materials with higher surface areas, enhanced activity and providing shape/size selectivity, zeolites are clear candidates for this reaction. This is due to thermal stability, flexibility in their chemical composition with the possibility of accommodating different metals in its framework. An important breakthrough in the field of zeolites occurred with the synthesis of titanium silicalite (TS1) [7], which was able to carry out the oxidation of different organic compounds with $\mathrm{H}_{2} \mathrm{O}_{2}$. However, TS- 1 presents geometric limitations to react larger molecules. Then, the synthesis of large pores zeolites 
started with the preparation of Ti-Beta initially by-post synthesis treatment [8] and later by isomorphous substitution of $\mathrm{Si}$ with $\mathrm{Ti}$ by direct synthesis $[9,10]$. Nevertheless, the catalytic properties of TiBeta were clearly worse than TS- 1 and the differences were attributed to the presence of $\mathrm{Al}$ in the framework, to a high density of connectivity defects (Si-OH groups giving hydrophilic properties) and to a higher acidity of framework Ti species. Afterward, an unseeded method in fluoride medium to obtain zeolite completely free of $\mathrm{Al}$ and connectivity defects was developed improving definitively the catalytic properties of Ti-Beta [11]. Nevertheless, despite the advances and the academic interest, the synthesis of Tizeolites in fluoride medium sets out different drawbacks such as long crystallization time and the use of hazardous fluoride that causes technical and environmental problems of corrosion and pollution.

From this point, different alternative strategies can be followed to improve the methodology. For example the use of lower toxic source of fluoride such as ammonium or seeds in order to decrease synthesis time. Other post-synthesis methods have been developed involving the incorporation of metal $\left(\mathrm{M}^{\mathrm{IV}}(\mathrm{Sn}, \mathrm{Ti}, \mathrm{Zr})\right)$ sites into the tetrahedral defects (sites) of modified beta zeolite framework. These routes start with the synthesis of Al or B beta zeolites, followed by the heteroatom removal and the creation of defects by acid treatments $[12,13]$. Then, the silanol groups generated can be used for anchoring the $\mathrm{M}^{\mathrm{IV}}$ precursors. Then different strategies were followed to introduce $\mathrm{M}^{\mathrm{IV}}$ such as chemical vapor deposition, solidstate mixing (grinding) [16], grafting at solvent reflux [17], or wetness impregnation [18] with adequated $\mathrm{M}^{\mathrm{IV}}$ containing reactants.

Another parameter of paramount importance is the presence of alkali ions. Indeed, the presence of high content of alkali ion during the synthesis of TS-1, Ti-Beta, and Sn-MFI constitutes an impediment to the crystallization of pure phase, precipitating metal oxide [19, 20]. Moreover, it was shown that alkali ions modify negatively the catalytic performance of zeolites for oxidation process [21, 22]. In contrary, other studies showed the positive effect of the addition of small amount of alkali ions such as for the epoxidation with $\mathrm{H}_{2} \mathrm{O}_{2}$ over TS-1, an increase of epoxide selectivity was observed and attributed to the neutralization of acid sites. Another experimental and computational work showed that the enhancement of catalytic performance for epoxidation could be attributed to weak cation- $\pi$ interactions between heavy alkali metal cations and adjacent olefin molecules improving their diffusion within the micropores. In contrary, light alkali metal cations difficult the diffusion because of stronger cation- $\pi$ interaction [23]. In this way, recent papers report the positive effect of the controlled presence of alkali ions in Sn-

Table 1. ICP analysis of post-synthesised Ti-Beta samples and DeAl-Beta.

\begin{tabular}{cccccccc}
\hline Samples & Li\% & Na\% & Ti\% & Si\% & Al\% & Si/Al & Si/Ti \\
\hline deAl-Beta & - & - & - & 41.06 & 0.02 & 1972 & - \\
Ti-Beta-PS & - & - & 1.68 & 44.56 & 0.02 & 2675 & 45 \\
Ti-Beta-PS-HT & - & - & 1.71 & 40.49 & 0.015 & 2778 & 40 \\
Ti-Beta-PS-HT-Na & - & 0.1 & 1.68 & 44.57 & 0.02 & 2676 & 45 \\
Ti-Beta-PS-HT-Li & 0.04 & - & 1.89 & 45.74 & 0.02 & 2585 & 41 \\
\hline
\end{tabular}

zeolites added in the reaction medium or for synthesis or postsynthesis treatment over their catalytic performance for sugars isomerization [24] and epimerization [25] or the conversion of sucrose into methyl lactate.

In this paper, we reported a convenient and optimized post-synthetic route for the preparation of Ti-Beta zeolite in where it has been successfully incorporated Lewis acid sites into the vacant tetrahedral (T)-sites of a dealuminated Beta-framework by ball-milling solidstate ion-exchange. A tribology-ball milling process was used in order to increase the interaction between dealuminated-Beta zeolite and Ti-precursor. Then, after consecutive processes of thermal treatment and calcination thanks to reversible hydrolysis of Ti-O-Si bonds and condensation steps we modified the coordination and so the nature of Lewis acid Ti centres. Moreover, partial cation exchange with alkali metal allowed to match the catalytic properties of Ti-Beta. Therefore, following this procedure it is possible to prepare optimized Ti-beta catalyst where $\mathrm{Ti}$ was tetrahedrally coordinated within the zeolite framework, with excellent activity as Lewis acid catalyst and stability for the isomerization of $\alpha$-pinene oxide into campholenic aldehyde in both batch and fixed bed reactor systems. Additionally, the catalytic performance of post-synthesised Ti-Beta was compared to a Ti-beta prepared in fluoride media.

\section{Results and discussion}

\subsection{Catalysts characterisation}

The preparation of Ti-Beta post-synthesis zeolite (Ti-Beta-PS) started by dealumination of a readily available commercial Al-Beta zeolite $\left(\mathrm{Si} / \mathrm{Al}=13\right.$ ) with an treatment in aqueous solution of $\mathrm{HNO}_{3}$ (see experimental). The ICP analysis demonstrated that $\mathrm{Al}$ was removed from the original material (Table 1) and the XRD pattern showed no structural changes in the Beta zeolite sample (Figure S1). From FT-IR spectroscopy it was seen that Al-Beta zeolite exhibited two characteristic bands of Brønsted acid sites (BAS) at $3610 \mathrm{~cm}^{-1}$ due to the presence of proton as cation of compensation of the negative charge associated to $\mathrm{Al}(\mathrm{Si}-\mathrm{O}(\mathrm{H})-\mathrm{Al})$, and isolated silanol groups at $3740 \mathrm{~cm}^{-1}$ (Figure S2). The FT-IR spectra of dealuminated Beta zeolite sample only registered the band at $3740 \mathrm{~cm}^{-1}$ supporting the removal of Al tetrahedrally coordinated. In a second step, the $\mathrm{Ti}$ atoms were incorporated in the vacant through solid state ion exchange by ball milling of dealuminated zeolite and $\mathrm{Cp}_{2} \mathrm{TiCl}_{2}$ (see experimental). After calcination at $550{ }^{\circ} \mathrm{C}$ in order to remove organic species and to complete the incorporation of $\mathrm{Ti}$ into the Beta zeolite framework, the DRUV-Vis spectrum obtained for TiBeta-PS sample shows broad and poorly defined absorption band in the $205-220 \mathrm{~nm}$ region that could indicate the formation of multiple metal species (Figure S3). Nevertheless, no indication of absorbance at 330-350 nm was observed, suggesting low presence of $\mathrm{TiO}_{2}$ octahedrally coordinated. Moreover, the plotted UV-Vis spectrum seem similar to those reported [26]. These data allow to conclude 
a)

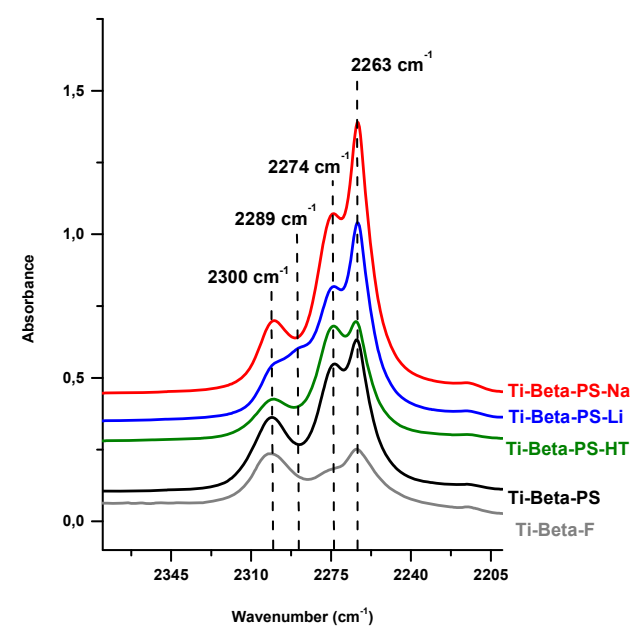

b)

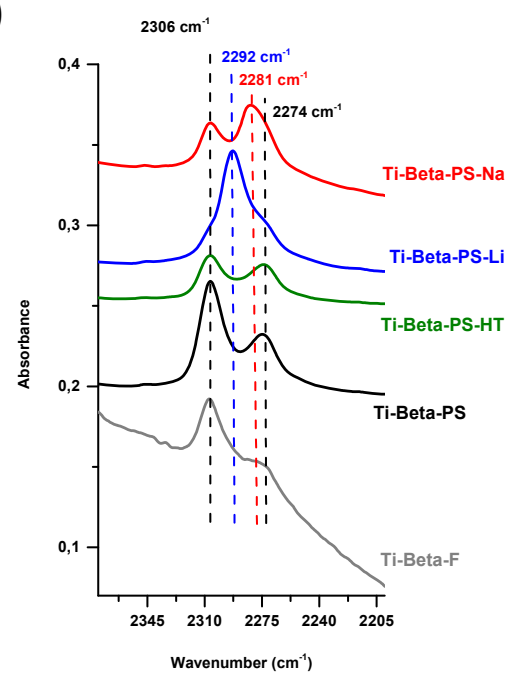

Figure 1. $\mathrm{CD}_{3} \mathrm{CN}$ adsorption/desorption profile for the different Ti-Beta-PS samples, (a) maximum coverage and (b) after desorption for 4 minutes.

that prepared sample contained isolated metal species and tetrahedrally coordinated within the zeolitic framework, and probably no bulk oxides. Finally, the preservation of crystallinity after the steps of dealumination and ball-milling for the incorporation of Ti was confirmed from the XRD (Figure S4).

In order to study the effect of the presence of alkali ions over the catalytic performance of Ti-Beta zeolite, we prepared exchanged $\mathrm{Na}$ and Li Ti-Beta-PS samples. For this, Ti-Beta-PS sample was treated with aqueous solution of $\mathrm{LiNO}_{3}$ and $\mathrm{NaNO}_{3}$ at $80^{\circ} \mathrm{C}$ for $15 \mathrm{~h}$ (see experimental). After calcination, similar DRUV-Vis spectra obtained for Ti-Beta-PS were registered with Ti-Beta-PS-HT, Na and Li samples show absorption in the $205-220 \mathrm{~nm}$ ultraviolet region that could indicate the formation of isolated metal species while the no

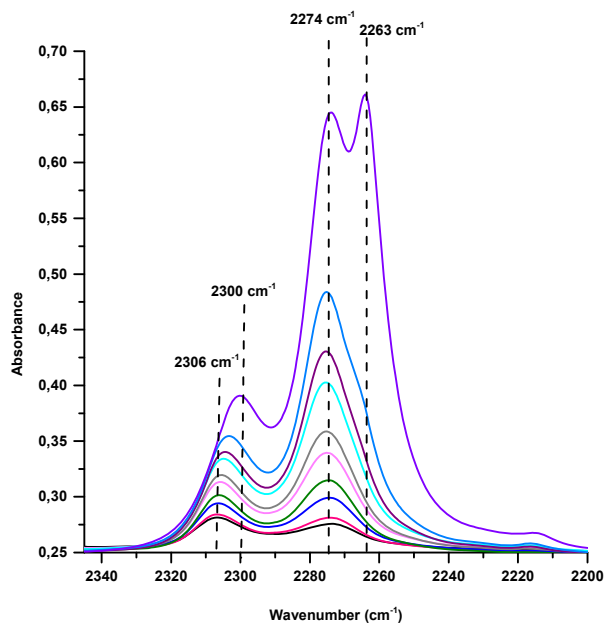

Figure 2. In situ $\mathrm{CD}_{3} \mathrm{CN}$ desorption profile for Ti-Beta-PS-HT. $\mathrm{CD}_{3} \mathrm{CN}$ was desorbed under a dynamic vacuum at room temperature. indication of absorbance at 330-350 $\mathrm{nm}$ suggested the absence of $\mathrm{TiO}_{2}$ (Figure S3). ICP analysis (Table 1 ) confirms the presence of $\mathrm{Na}$ and $\mathrm{Li}$ in the treated samples as well as that $\mathrm{Ti}$ wt\% content remained constant. More especially, Li and $\mathrm{Na}$ content was of $0.04 \%$ and $0.1 \%$ respectively, i.e a 13 and $10 \mathrm{~mol} \%$ respect to Ti, respectively.

To obtain more information about of titanium speciation within TiBeta-PS samples as synthesised and after thermal treatment with $\mathrm{NaNO}_{3}$, LiNO 3 , FT-IR $\mathrm{CD}_{3} \mathrm{CN}$ absorption was performed. When deuterated acetonitrile was adsorbed on the different calcined TiBeta samples, several bands in the $2260-2310 \mathrm{~cm}^{-1}$ IR region associated with $\mathrm{v}(\mathrm{C} \equiv \mathrm{N})$ stretching vibration were observed. The adsorption band at $\pm 2263 \mathrm{~cm}^{-1}$ corresponds to weakly physisorbed $\mathrm{CD}_{3} \mathrm{CN}$ and disappeared at the maximum of desorption (Figure $1 \mathrm{~b}$ ). There was a noticeable shoulder at $\pm 2274 \mathrm{~cm}^{-1}$ that was attributed to $\mathrm{CD}_{3} \mathrm{CN}$ coordinated to $\mathrm{Br} \varnothing$ nsted acid sites likely due to the silanol species that were present in the materials. Following desorption under dynamic vacuum for various time periods, it is clear that the weakly-bound physisorbed $\mathrm{CD}_{3} \mathrm{CN}$ was first removed, followed by the $\mathrm{CD}_{3} \mathrm{CN}$ species bound to the Brønsted acid sites (Figure 2). The presence of silanol groups was supported by the FTIR spectra of activated Ti-Beta zeolites in the $\mathrm{OH}$ stretching region. Indeed, it was observed that post-synthesis samples present a large number of silanol groups as it could be expected due to the methodology of the procedure since the dealumination as well as the incorporation of $\mathrm{Ti}$ atoms via a solid state ion-exchange by ball milling induced silicon coordination defect. Then the two bands at \pm 3736 and $\pm 3746 \mathrm{~cm}^{-1}$ were assigned to terminal $\mathrm{Si}-\mathrm{OH}$ groups (Figure 3 ). The difference in wavenumbers was primarily related to the silanol groups being involved or not in weak hydrogen bonding. Thus, the band at \pm 3743 $\mathrm{cm}^{-1}$ was assigned to free $\mathrm{Si}-\mathrm{OH}$ groups [27], at $\pm 3736 \mathrm{~cm}^{-1}$ being assigned to those $\mathrm{Si}-\mathrm{OH}$ groups in which the $\mathrm{O}$ is weakly hydrogen bonded to an adjacent $\mathrm{OH}$ moiety [28]. These acidic $\mathrm{Si}-\mathrm{OH}$ groups were partially or totally passivated by post-synthesis modification with alkali cations. From both spectra, it is concluded that post- 
a)

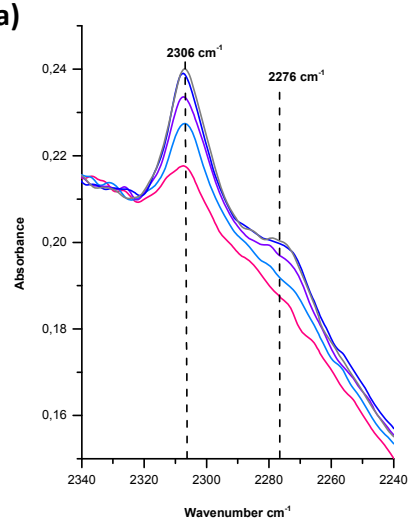

d) b)

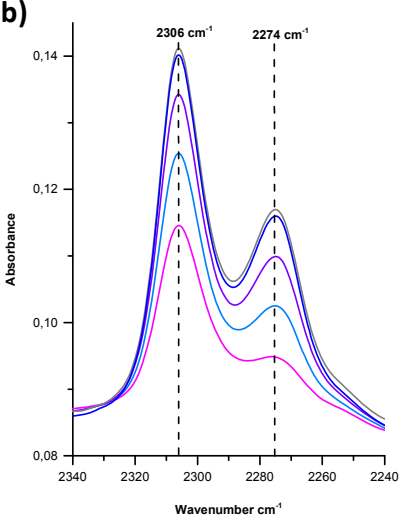

c)

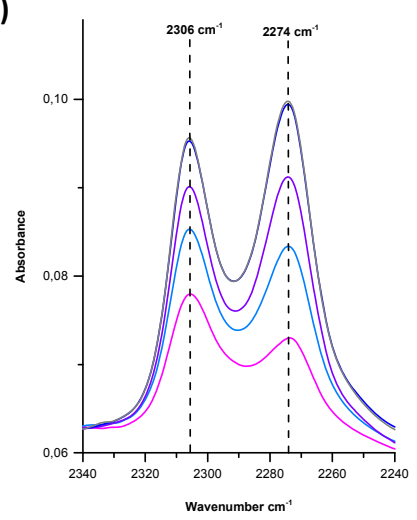

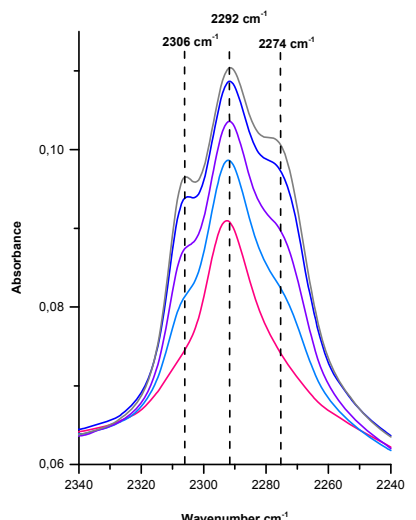

e)

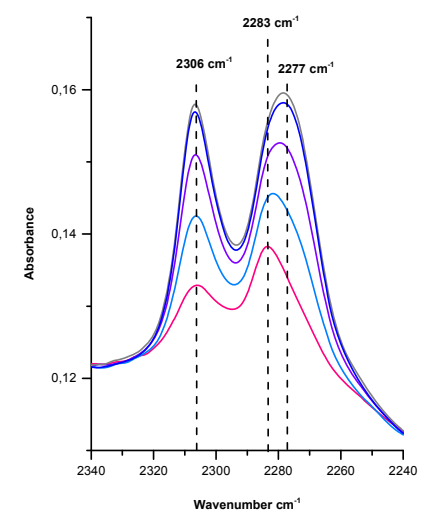

Figure 3. IR spectra at increasing acetonitrile coverage (1.9 mBar), on (a) Ti-Beta-PS, (b) Ti-Beta-PS-HT, (c) Ti-Beta-PS-HT-Na, (d) Ti-BetaPS-HT-Li and (e) Ti-Beta-F samples.

synthesised Ti-Beta samples present a larger density of silanol groups than Ti-Beta synthesised in fluoride media practically without defect of coordination.

$\mathrm{CD}_{3} \mathrm{CN}$ adsorption bands at \pm 2306 and $\pm 2300 \mathrm{~cm}^{-1}$ were also observed and corresponds to coordinated $\mathrm{CD}_{3} \mathrm{CN}$ onto the Tilv Lewis acid centres [20]. Considering the IR spectra at increasing acetonitrile low coverage over the different samples, initially $\mathrm{CD}_{3} \mathrm{CN}$ adsorption band

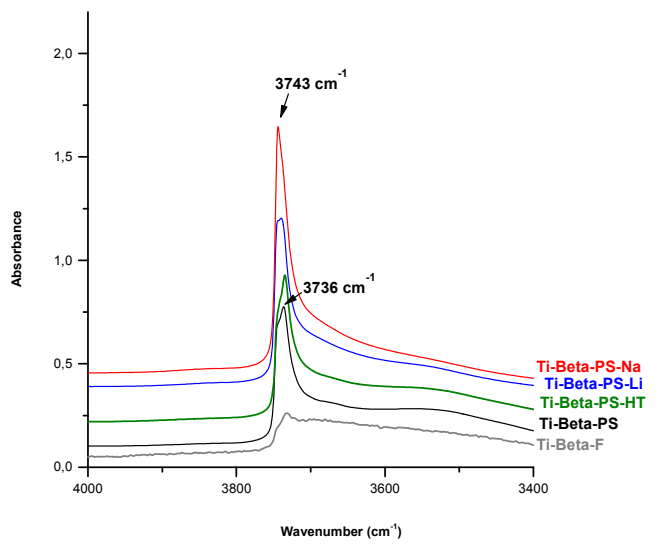

Figure 4. IR spectra in the $\mathrm{OH}$ stretching region for the different Ti-Beta-PS samples after activation. at $\pm 2306 \mathrm{~cm}^{-1}$ (Figure 3) and at higher coverage $\mathrm{CD}_{3} \mathrm{CN}$ adsorption band at $\pm 2300 \mathrm{~cm}^{-1}$ were observed (Figure 1 (a) and Figure S5). Therefore, if we take into accounts studies of IR $\mathrm{CD}_{3} \mathrm{CN}$ absorption on Sn-Beta [29] and by analogy we assume that the fact that there are two bands indicate that two types of Lewis acids sites (LAS) are present. Moreover, the higher relative shift of the band and the fact that the adsorption initiates at $2306 \mathrm{~cm}^{-1}$, allow to suggest that Lewis acid sites at $2306 \mathrm{~cm}^{-1}$ interacts more strongly with acetonitrile than the other at $2300 \mathrm{~cm}^{-1}$. Indeed, in the case of Sn-Beta, partially

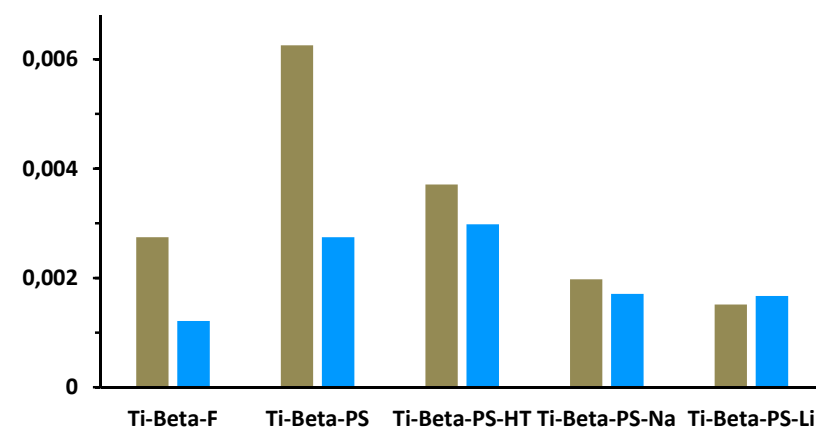

Figure 5. Comparison after normalisation (sample weight) of the $\mathrm{CD}_{3} \mathrm{CN}$ absorbance on Ti-Lewis acid site at $(-) 2308 \mathrm{~cm}^{-1}$ and weak Brønsted acid SiO-H groups at (") $2274 \mathrm{~cm}^{-1}$. 
Table 2. Effect of metal Lewis acid centre.

\begin{tabular}{cccccccccc}
\hline \multirow{2}{*}{ Catalyst } & \multirow{2}{*}{ Conv. $\alpha-P O \%$} & \multirow{2}{*}{ CA Sel.\% } & \multirow{2}{*}{ TON* } & \multicolumn{5}{c}{ Yield \% } \\
\cline { 5 - 9 } & & & & CA & 3-Pinanone & Iso-CA & Carveol & Pinocarveol \\
\hline deAl-Beta & 28.1 & 47.4 & - & 13.3 & 0.8 & 1.9 & 3.0 & 1.2 \\
Ti(1.9\%)-Beta-F & 93.7 & 82.1 & 3718 & 76.9 & 1.8 & 2.3 & 3.2 & 0.1 \\
Ti(1.7\%)-Beta-PS & 95.3 & 59.8 & 3248 & 57.0 & 4.0 & 5.4 & 8.5 & 0.4 \\
Zr(1.9\%)-Beta-F & 93.1 & 26.3 & 2281 & 24.5 & 28.0 & 13.1 & 9.2 & 0.6 \\
Sn(1.9\%)-Beta-F & 99.1 & 17.3 & 2050 & 17.2 & 29.6 & 29.0 & 7.9 & 0.3 \\
\hline
\end{tabular}

Reaction conditions: Reaction conditions: microwave heating, 150으, 25mg of zeolite, $2 \mathrm{mmol}$ of $\alpha-\mathrm{PO}, 760 \mathrm{mg}$ of acetonitrile and 3 min of reaction time. ${ }^{*}$ TON was calculated as the number of moles of CA produced by the number of mole of metal as active sites.

hydrolysed and nonhydrolysed framework Sn sites, or open-closed sites, were identified and attributed to adsorption bands at \pm 2308 and $\pm 2316 \mathrm{~cm}^{-1}$, respectively. In the case of titanosilicates, reported detailed EXAFS studies of TS-1 allowed to distinguish between the tetrapodal and tripodal $\left(\mathrm{Ti}(\mathrm{OSi})_{3} \mathrm{OH}\right)$ structures [30]. Similarly, the bands at \pm 2300 and $\pm 2306 \mathrm{~cm}^{-1}$ are assigned to open-closed Ti-Lewis acid centres.

Additionally, for Ti-Beta-PS-HT-Li a new band at $\pm 2292 \mathrm{~cm}^{-1}$ is detected (Figure 1 and Figure 3 (d)). In comparison to IR spectra of $\mathrm{CD}_{3} \mathrm{CN}$ on Sn-Beta-Li reported by Tatsumi and co-workers [31] this band is assigned to $\mathrm{CD}_{3} \mathrm{CN}$ adsorption on $\mathrm{Li}$ cations that can act as Lewis acid sites. When we compare the minimum acetonitrile coverage spectra of the five Ti-Beta samples (Figure $3 \mathrm{a}, \mathrm{b}, \mathrm{c}, \mathrm{d}$ and e, Figure 4 ) it is noticeable that Ti-Beta-F and Ti-Beta-PS samples present different density of Lewis acid sites and of silanol groups. This tendency is illustrated in Figure 5 where after normalization (wafer weight of zeolite sample), $\mathrm{CD}_{3} \mathrm{CN}$ absorbance heights at \pm 2306 and $\pm 2274 \mathrm{~cm}^{-1}$ determined after deconvolution of the IR spectra with the ORIGIN software program (Figure S6) were plotted. It is clear that Ti-Beta-PS sample presents a higher number of strong Ti-Lewis acid sites than other Ti-Beta samples and that through post synthesis thermal treatment and cation exchange the population of strong TiLewis acid sites (Ti-LAS) and of silanol groups were modified and decreased. In the case of Ti-Beta-PS-Li sample, the $\mathrm{CD}_{3} \mathrm{CN}$ adsorption over $\mathrm{Li}$ induces an overlapping of the band at $\pm 2292 \mathrm{~cm}^{-1}$ together with the band at $2306 \mathrm{~cm}^{-1}$ (Figure 1 (b) and Figure S6 (b)). The lower shift of the bands indicates that Li can acts as weaker Lewis acid sites than $\mathrm{Ti}$, while the changes in the intensity of the band at $\pm 2306 \mathrm{~cm}^{-1}$ indicates a weakening of Ti-Lewis acid sites respect to IR $\mathrm{CD}_{3} \mathrm{CN}$ spectra of Ti-Beta-PS and Ti-Beta-PS samples. Tatsumi and coworkers showed that the weakening of Sn-Lewis acid sites was enhanced by increasing the size of the cations from Li to Cs, and that the more adequate cation to maintain the original Lewis acidity on the $\mathrm{Sn}$ atoms was Li. Different works reported that the incorporation of alkali metal occurred through cation exchange with proton of SiO$\mathrm{H}$ close to $\mathrm{Sn}$ and not on SnO-H [25, 31]. Moreover, that cation exchange takes place indifferently on both closed and open Sn sites through dynamic hydrolysis of $\mathrm{Si}-\mathrm{O}-\mathrm{Sn}$ bonds during aqueous treatment. In the case of Ti-Beta-PS-HT-Na a new band at $\pm 2282 \mathrm{~cm}^{-}$ ${ }^{1}$ is detected indicating that adsorption on $\mathrm{Na}$ was weaker than on $\mathrm{Li}$ (Figure 1 and Figure 3 (c)) while both bands at \pm 2306 and $\pm 2300 \mathrm{~cm}^{-}$ ${ }^{1}$ were maintained, indicating that Lewis acidity of Ti-atoms was preserved.

These data of characterisation showed that with a small amount of $\mathrm{Li}$ or $\mathrm{Na}$, partial cation exchange on SiO-H close to Ti atoms occurred, passivating Brønsted acidity of adjacent silanol groups and maintaining the Lewis acidity of Ti-atoms similarly to what was described with Sn-Beta zeolites but modifying both density of TiLewis acid and weak Brønsted acid sites.

\subsection{Catalytic activity tests}

\section{Reaction in sealed vial under microwave}

At first, the catalytic tests of the titanosilicate catalysts herein prepared for the $\alpha$-pinene oxide isomerization were performed in sealed vial under microwave irradiation. The benefits of microwave heating over conventional ovens are well known, such as higher rate of reaction, better yield and higher selectivity (less formation of side product), high reproducibility thanks to the homogeneity of microwave irradiation and finally a lower energy usage. In Table 2, the catalytic activity of different Beta samples prepared conventionally in fluoride medium and incorporating different metal with Lewis acid properties such as $\mathrm{Ti}, \mathrm{Sn}$ and $\mathrm{Zr}$ were compared. Because of the high reactivity of $\alpha$-pinene oxide different isomerization products were formed and detected such as carveols, iso-campholenic aldehyde, 3-pinanone and pinocarveol (Scheme 1). As it has been reported in the bibliography, the more adequate metal

Table 3. Effect of hydrothermal treatment over the catalytic performance of post-synthesized Ti-beta.

\begin{tabular}{ccccccccc}
\multirow{2}{*}{ Entry } & \multirow{2}{*}{ Catalyst } & \multirow{2}{*}{ Conv.\% } & \multicolumn{5}{c}{ Yield \% } & CA \\
\cline { 4 - 6 } & & & CA & 3-Pinanone & Iso CA & Pinocarveol & Carveol & Sel. \% \\
\hline 1 & Ti-Beta-F & 93.7 & 76.9 & 1.8 & 2.3 & 0.1 & 3.2 & 82.1 \\
2 & Ti-Beta-PS & 95.3 & 57.0 & 4.0 & 5.4 & 0.4 & 8.5 & 59.8 \\
3 & Ti-Beta-PS-HT & 97.2 & 79.9 & 1.9 & 1.9 & 0.2 & 3.8 & 82.2 \\
4 & Ti-Beta-PS-HT-Li(0.04\%) & 99.1 & 86.5 & 1.5 & 1.4 & 0.1 & 2.6 & 87.3 \\
5 & Ti-Beta-PS-HT-Na(0.1\%) & 97.1 & 83.3 & 1.5 & 1.3 & 0.6 & 2.5 & 85.8 \\
\hline
\end{tabular}

Reaction conditions: Reaction conditions: microwave heating, $150^{\circ} \mathrm{C}, 25 \mathrm{mg}$ of zeolite, $2 \mathrm{mmol}$ of $\alpha-\mathrm{PO}, 760 \mathrm{mg}$ of acetonitrile and 3 min of reaction time. 
Table 4. Effect of addition $\mathrm{NaNO}_{3}$ or $\mathrm{LiNO}_{3}$ following different method.

\begin{tabular}{|c|c|c|c|c|c|c|c|c|}
\hline \multirow{2}{*}{ Entry } & \multirow{2}{*}{ Catalyst } & \multirow{2}{*}{$\begin{array}{c}\alpha-\mathrm{PO} \\
\text { Conv.\% }\end{array}$} & \multicolumn{3}{|c|}{ Yield\% } & \multirow{2}{*}{$\begin{array}{c}\text { CA } \\
\text { Sel.\% }\end{array}$} & \multirow{2}{*}{$\begin{array}{c}\text { Cation } \\
\%\end{array}$} & \multirow{2}{*}{ Method } \\
\hline & & & CA & Pinanone & Iso-CA & & & \\
\hline 1 & Ti(1.9\%)-Beta-PS-HT & 95.6 & 81.6 & 2.7 & 2.5 & 85.3 & $0.00 \%$ & - \\
\hline 2 & $\begin{array}{c}\text { Ti(1.9\%)-Beta-PS- } \\
\text { Li(0.04\%) }\end{array}$ & 99.8 & 88.6 & 2.4 & 2.3 & 88.8 & $0.04 \%$ & Li-reflux \\
\hline 3 & Ti-Beta-PS-HT-Li-1 & 93.9 & 77.4 & 1.8 & 1.9 & 82.4 & $0.01 \%$ & Li-impregnation \\
\hline & Ti-Beta-PS-HT-Li-2 & 86.3 & 72.5 & 2.1 & 1.9 & 84.0 & $0.03 \%$ & Li-impregnation \\
\hline 4 & Ti-Beta-PS-HT-Li-3 & 84.8 & 70.9 & 2.0 & 1.7 & 83.7 & $0.06 \%$ & Li-impregnation \\
\hline 5 & Ti-Beta-PS-HT-Li-4 & 93.5 & $77.1 \%$ & 2.3 & 2.1 & 82.5 & $0.03 \%$ & Li-solution \\
\hline 6 & Ti-Beta-PS-HT-Na(0.04\%) & 99.2 & $83.6 \%$ & 2.4 & 2.3 & 84.3 & $0.04 \%$ & Na-reflux \\
\hline 7 & Ti-Beta-PS-HT-Na-1 & 96.7 & $83.2 \%$ & 2.0 & 2.1 & 86.1 & $0.02 \%$ & Na-impregnation \\
\hline
\end{tabular}

Reaction conditions: microwave heating, $150 \circ \mathrm{C}, 8 \mathrm{mg}$ of zeolite, $2 \mathrm{mmol}$ of $\alpha-\mathrm{PO}, 380 \mathrm{mg}$ of acetonitrile and $8 \mathrm{~min}$ of reaction time.

to perform the isomerization of $\alpha-\mathrm{PO}$ was Ti. Under the reactions conditions, CA was obtained with yield and selectivity up to 76.9 and 82.1\%, after 3 min of reaction time and the calculated TONs allowed to order the catalytic performance of the different metal-zeolite samples to produce CA as follows: Ti-Beta-F>Ti-Beta-PS>Zr-Beta$\mathrm{F}>\mathrm{Sn}$-Beta-F (Table 2). As we mentioned above the preparation Beta samples incorporating $\mathrm{Ti}, \mathrm{Zr}$ or $\mathrm{Sn}$ in fluoride media involves several drawbacks and so pushed to research and develop alternative routes of synthesis. Likewise we studied the preparation of Ti-Beta zeolite following a post-synthesis method based on the dealumination of Albeta commercial sample and the posterior incorporation of $\mathrm{Ti}$ in the tetrahedral defects generated through solid state ion-exchange process using a ball-mill. It has been reported than only tetrahedrally and 5-coordinated Ti species acted as Lewis acid active sites and that extra-framework species with octahedral coordination were inactive species [32, 33]. From characterisation data we showed the successful incorporation of $\mathrm{Ti}$ in tetrahedrally coordinated position. In Table 3, the results obtained over the different Ti-beta samples following conventional method in fluoride medium and, following a post-synthesis method are reported. The results of Table 3, Entries 1 and 2 showed that Ti-Beta-PS exhibited notably lower selectivity to CA than Ti-Beta-F. Additionally, the catalytic performance of the dealuminated Beta sample (deAl-Beta) was explored. As it could be expected, deAl-Beta exhibited low catalytic activity and CA selectivity. Considering the route of preparation of the Ti-Beta-PS sample and the IR characterisation data, this result was attributed to the coordination defects created during the incorporation of Ti into the Beta framework and so to the presence of high density of silanol groups with weak Brønsted acidity, providing distinct active centres that exhibit diverse activity and selectivity. In order to improve the catalytic performance of the Ti-Beta sample, we envisioned that consecutive processes of thermal treatment and calcination thanks to reversible hydrolysis of Ti-O-Si bonds and condensation steps could modify the coordination and so the nature of Lewis acid $\mathrm{Ti}$ centres (partially hydrolysed and nonhydrolysed). As well as, a study of cation exchange with alkali metal can allow us to match the catalytic properties of Ti-Beta inhibiting the weak Brønsted acidity of adjacent silanol groups to Ti-atoms on the one hand and adjust the Ti-Lewis acid strength on the other hand. For this, Ti-Beta-PS was treated with different aqueous solutions of $\mathrm{LiNO}_{3}, \mathrm{NaNO}_{3}$ and water at $85 \circ \mathrm{C}$ for $15 \mathrm{~h}$. After calcination, the catalytic performance exhibited by the three Ti-Beta-PS samples submitted to hydrothermal treatment were notably improved (Table 3, entries 35). Interestingly, after a simple hydrothermal treatment at $85 \circ \mathrm{C}$ and calcination, the catalytic performance of Ti-Beta-PS was considerably improved since the CA selectivity increased from 59.8 up to $82.2 \%$. This catalytic results were similar to ones achieved in the presence of Ti-Beta-F. From Figure 5, it was noticeable that Ti-Beta-PS presented the highest density of strong Lewis acid sites as well as of weak Brønsted acid sites (SiO-H) (BAS). However, after hydrothermal treatment Ti-Beta-PS-HT has lower density of Ti-LAS than Ti-Beta-PS but similar to Ti-Beta-F, while the density of BAS are similar for both samples Ti-Beta-PS and Ti-Beta-PS-HT and higher than Ti-Beta-F. When the Ti-Beta-PS catalytic behaviour was checked after the hydrothermal treatment, with $\mathrm{NaNO}_{3}$ and $\mathrm{LiNO}_{3}$ solutions and calcination steps, the results showed that it is possible to increase newly the CA selectivity up to $87.3 \%$. Now, the achieved CA selectivity is higher than one exhibited in the presence of Ti-Beta-F.

Table 5. Effect of nature of solvent over the catalytic performance of post-synthesized Ti-Beta-PS-HT-Na(0.1\%).

\begin{tabular}{cccccccc}
\hline \multirow{2}{*}{ Solvent } & \multirow{2}{*}{ Conv. $\alpha-P O \%$} & \multirow{2}{*}{ CA Sel.\% } & \multicolumn{5}{c}{ Yield \% } \\
\cline { 5 - 8 } & & & CA & 3-Pinanone & Iso-CA & Carveol & Pinocarveol \\
\hline ACN & $93.2 \%$ & $83.4 \%$ & $77.8 \%$ & $2.4 \%$ & $2.1 \%$ & $2.7 \%$ & $0.5 \%$ \\
Toluene & $87.0 \%$ & $86.2 \%$ & $75.0 \%$ & $5.3 \%$ & $2.9 \%$ & $1.8 \%$ & $0.1 \%$ \\
DMA & $9.4 \%$ & $78.9 \%$ & $7.4 \%$ & $0.4 \%$ & $0.7 \%$ & $1.5 \%$ & $0.2 \%$ \\
MeCyHex. & $83.5 \%$ & $78.7 \%$ & $65.7 \%$ & $4.3 \%$ & $2.7 \%$ & $2.1 \%$ & $0.9 \%$ \\
\hline
\end{tabular}

Reaction conditions: Reaction conditions: microwave heating, $150 \circ \mathrm{C}, 8 \mathrm{mg}$ of zeolite, $2 \mathrm{mmol}$ of $\alpha-\mathrm{PO}, 0.485 \mathrm{~mL}$ of solvent and 8 min of reaction time. 
Additionally the CA selectivity, $\alpha-P O$ conversion and kinetic constant were plotted versus the height of Lewis acid sites at $2306 \mathrm{~cm}^{-1}$ and Brønsted acid sites at $2274 \mathrm{~cm}^{-1}$. The Figure S7 illustrates that a correlation between the height of Lewis acid sites and CA selectivity and kinetic constant was established, decreasing the CA selectivity as well as kinetic constant with an increase of the height (number of Lewis active sites). Nevertheless, when the CA selectivity and kinetic constant were plotted versus the height of Brønsted acid sites no trend could be established. These results showed that the strength and number of Lewis active is a key factor to maximise the CA selectivity since the $\mathrm{Li}$ and $\mathrm{Na}$ cation exchange induced and a weakening in the Ti-Lewis acidity maximising CA selectivity. In the meantime, $\alpha$-PO conversion remained constant regardless of the height of Lewis acid sites or Brønsted acid sites.

IR study of $\mathrm{CD}_{3} \mathrm{CN}$ adsorption allowed to conclude that $\mathrm{Li}$ and $\mathrm{Na}$ cation were exchanged by proton of $\mathrm{SiO}-\mathrm{H}$ group adjacent to $\mathrm{Ti}$ atom, partially hydrolysed or nonhydrolysed since treatment was performed in presence of water and that hydrolysis of Si-O-Ti occurred. In this way, the weak Brønsted acidity of silanol groups can be controlled and partially inhibited. Moreover, the alkali cation ( $\mathrm{Li}$ and $\mathrm{Na}$ ) not only exhibit Lewis-acidity but also induced a weakening in the Ti-Lewis acidity. These catalytic results showed that the catalytic properties of Ti-Beta-PS were modified through alkali cations exchange reducing side reactions and maximising $\alpha-P O$ conversion and CA selectivity. Then characterisation data together with catalytic performance of the different Ti-Beta samples let to conclude that Ti-Lewis acid strength was softened and tailored thank to the Li or $\mathrm{Na}$ cation exchange and the weak Brønsted acidity of $\mathrm{Ti}$ atoms adjacent $\mathrm{SiO}-\mathrm{H}$ was passivated, maximising the catalytic properties of Ti-Beta sample to the production of CA.

In order to determine if there exist a maximum in the $\mathrm{Na}$ and $\mathrm{Li}$ content, a study of addition of $\mathrm{Li}$ and $\mathrm{Na}$ following different procedures was performed, by impregnation or by adding the alkali in solution in methanol (Table 4), either to the Ti-Beta catalyst or added directly to the reaction medium. The results were compared to hydrothermal treatment (Reflux). The addition of $\mathrm{Li}$ by impregnation or in methanol solution did not improved the catalytic performance of the Ti-Beta-PS-HT obtained by post-synthesis method and submitted to hydrothermal treatment at $85^{\circ} \mathrm{C}$ for $15 \mathrm{~h}$, and on the contrary, we could observe a slight decrease of the catalytic results (Table 4, Entries 2-5). In the case of $\mathrm{Na}$, a slight increase of the catalytic activity of the Ti-Beta samples was observed (Table 4, Entries 6 and 7).

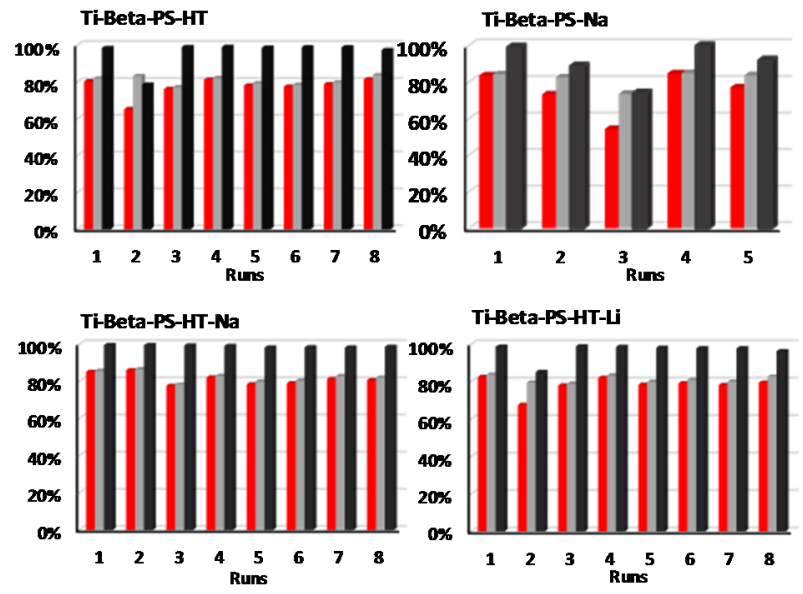

Figure 6. Reaction conditions: Reaction conditions: microwave heating, $150^{\circ} \mathrm{C}, 8 \mathrm{mg}$ of zeolite, $2 \mathrm{mmol}$ of $\alpha-P O, 380 \mathrm{mg}$ of acetonitrile and $8 \mathrm{~min}$ of reaction time. $\bullet \mathrm{CA}$ yield, $\bullet \mathrm{CA}$ selectivity, $\bullet \alpha$-PO conversion.

Then the stability of the catalytic activity of the different Ti-Beta samples was studied. For this purpose, different Ti-Beta catalysts were reused up to 8 consecutive runs. After each cycle, the solid catalyst was separated by filtration, washed with acetonitrile and calcined in air at $550 \circ$. Figure 6 showed that the different samples exhibited a stable catalytic behaviour along the different runs and that exchanged-alkali metal samples exhibited higher catalytic performance than Ti-Beta-HT. Noticeably, these results demonstrated that alkali metal was strongly exchanged and stabilized. In terms of yield and selectivity, Ti-Beta-PS-HT-Na(0.1\%) exhibited slightly higher performance. So, we considered that TiBeta-PS-Na(0.1\%) exhibited the more adequate catalytic properties. In order to consider the nature of Ti active sites of the used catalysts, FT-IR $\mathrm{CD}_{3} \mathrm{CN}$ absorption of the used Ti-Beta-PS-HT, Ti-Beta-PS-HT-Na and Ti-Beta-PS-HT-Li beta samples, after calcination at 550ㄷ for $5 \mathrm{~h}$, was performed. For both adsorption and desorption profiles of the used Ti-Beta-PS-HT, Ti-Beta-PS-HT-Na and Ti-Beta-PS-HT-Li samples, the same characteristic bands described for the original samples are observed. For adsorption (Figure S8 (a)), the band at $\pm 2263 \mathrm{~cm}^{-1}$ corresponding to weakly physisorbed $\mathrm{CD}_{3} \mathrm{CN}$ is lower for all postsynthesised samples respect to original ones. A shoulder at \pm 2274 $\mathrm{cm}^{-1}$ attributed to $\mathrm{CD}_{3} \mathrm{CN}$ coordinated to Brønsted acid sites due to the presence of the silanol species as well as the band at $2300 \mathrm{~cm}^{-1}$ corresponding to coordinated $\mathrm{CD}_{3} \mathrm{CN}$ onto the Tiv Lewis acid centres

Table 6. Effect of $\alpha$-PO concentration over the catalytic performance of post-synthesized Ti-Beta-PS-HT-Na(0.1\%).

\begin{tabular}{|c|c|c|c|c|c|c|c|c|c|}
\hline \multirow{2}{*}{ Entry } & \multirow{2}{*}{$\alpha-P O$ wt $\%$} & \multirow[t]{2}{*}{$\alpha-P O \mathrm{~mol} / \mathrm{L}$} & \multirow{2}{*}{ Conv. $\alpha-\mathrm{PO} \%$} & \multirow{2}{*}{$\begin{array}{c}\text { CA } \\
\text { Sel.\% }\end{array}$} & \multicolumn{5}{|c|}{ Yield \% } \\
\hline & & & & & CA & 3-Pinanone & Iso-CA & Carveol & Pinocarveol \\
\hline 1 & 54.5 & 5.7 & 93.0 & 84.9 & 79.0 & 2.5 & 2.1 & 2.7 & 0.5 \\
\hline 2 & 47.8 & 4.1 & 93.2 & 83.4 & 77.8 & 2.4 & 2.1 & 2.7 & 0.5 \\
\hline 3 & 30.2 & 2.1 & 98.2 & 87.3 & 85.8 & 2.2 & 1.9 & 2.7 & 0.4 \\
\hline 4 & 18.2 & 1.0 & 96.0 & 89.0 & 85.5 & 1.9 & 1.6 & 1.9 & 0.1 \\
\hline
\end{tabular}

Reaction conditions: microwave heating, $150 \circ \mathrm{C}, 8 \mathrm{mg}$ of Ti-Beta-PS-HT-Na(0.1\%), $2 \mathrm{mmol}$ of $\alpha-\mathrm{PO}$, different volume of acetonitrile. Ti content: $2 \mathrm{wt} \%$. 
Table 7. Effect of catalyst amount over the catalytic performance of post-synthesized Ti-Beta-PS-HT-Na(0.1\%).

\begin{tabular}{ccccccccc}
\hline \multirow{2}{*}{ Time (min) } & \multirow{2}{*}{ wt\% } & $\begin{array}{c}\text { Conv. } \alpha- \\
\text { PO\% }\end{array}$ & CA Sel.\% & \multicolumn{5}{c}{ Yield \% } \\
\cline { 5 - 8 } & & & CA & 3-Pinanone & Iso-CA & Carveol & Pinocarveol \\
\hline 8 & 1.2 & 71.9 & 82.2 & 59.1 & 2.1 & 1.8 & 2.4 & 0.6 \\
4 & 2.3 & 93.2 & 83.4 & 77.8 & 2.4 & 2.1 & 2.7 & 0.5 \\
3 & 3.4 & 93.4 & 85.1 & 79.5 & 2.2 & 1.9 & 2.6 & 0.4 \\
2 & 5.1 & 99.4 & 84.8 & 84.2 & 2.3 & 2.0 & 2.8 & 0.4 \\
\hline
\end{tabular}

Reaction conditions: Reaction conditions: microwave heating, 4, 8, 12, 18 or $25 \mathrm{mg}$ of Ti-Beta-PS-HT-Na(0.1\%), $2 \mathrm{mmol}$ of $\alpha-\mathrm{PO}$, $375 \mathrm{mg}$ of acetonitrile.

are present similarly to original samples. At maximum desorption (Figure S8 (b)), similar profiles are observed for Ti-Beta-PS-HT and TiBeta-F samples with two bands at 2308 and $2274 \mathrm{~cm}^{-1}$ corresponding to closed Ti-Lewis acid centres and Brønsted acid sites, respectively. Additionally, for Ti-Beta-PS-HT-Li the band at $\pm 2292 \mathrm{~cm}^{-1}$ is detected. Different intensities of the different bands are observed, for the TiBeta-PS-HT-Li sample before and after reaction, indicating a subtle variation in the nature of the Lewis acid centers that nevertheless did not influence the catalytic activity of the sample since it can be reused without loss of selectivity or conversion (Figure 6). A similar subtle change in the bands intensity of the Ti-Beta-PS-HT-Na sample was observed at 2308 and $2281 \mathrm{~cm}^{-1}$. Also in this case the slight changes in the nature of the Lewis acid active sites did not affect the catalytic properties of the Ti-Beta-PS-HT-Na sample that could be reused several cycles without loss of selectivity or conversion. DRUVVis spectra obtained for Ti-Beta samples after their use as isomerization catalyst were similar to ones registered for fresh catalysts (Figure S9). A broad and poorly defined absorption band in the $205-220 \mathrm{~nm}$ region indicates the formation of multiple metal species and no indication of absorbance at 330-350 nm suggests low presence of $\mathrm{TiO}_{2}$ octahedrally coordinated. Then, the characterisation data of used Ti-Beta-PS samples allowed to confirm the stability of the active acid sites.

In chemical processes, it is important to select the optimum reactions conditions such as solvent (nature and amount) and temperature. More especially, in the case of solid catalysts where compete the coadsorption/desorption of solvent, reactant and products. Competitive adsorption/desorption determine the intra-porous concentration of reactant and products and can modify greatly the process selectivity and side reaction productions. In the case of $\alpha$-PO isomerization, different works reported the effect of polarity and basicity of the chosen solvents over the selectivity of the process [13]. As well as, non-polar solvent seemed to favour selective
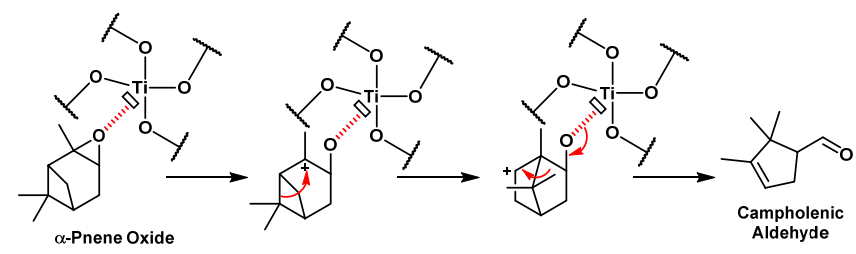

Scheme 2. Proposed mechanism for the isomerisation of $\alpha-\mathrm{PO}$ campholenic aldehyde while polar and basic solvents such as N,Ndimethylacetamide (DMA) allowed to increase the selectivity to carveols. Furthermore, in the case of Ti-Beta zeolite the synthesis procedure affects hydrophobic/hydrophilic properties of Ti-Beta controlled by the density of silanol groups. A sample without defects, i.e. with a reduced amount of silanol groups, will preferentially adsorb non-polar molecules over more polar ones. Thus, the catalytic results of the use of 4 different solvents were reported in the Table 5 using Ti-Beta-PS-HT-Na(0.1\%) as catalyst. We observed that at 150 ㅇ $\mathrm{C}$ the Ti-Beta-PS sample herein prepared was totally deactivated by the use of DMA, due to strong adsorption over active sites, being the conversion only of $9.4 \%$. The result was in disagreement with recently published paper where the use of DMA for $\alpha$-PO isomerization in the presence of Ti-MWW produced at 140ㄷ CA with selectivity close to $75 \%$ at $75 \%$ conversion of $\alpha$-PO. In the case of TiBeta-PS-HT-Na(0.1\%) the best selectivity were obtained with acetonitrile and toluene, up to $86 \%$, being higher the conversion in the presence of acetonitirile (93\%) which appears more suitable solvent.

Given that for the development of greener process and the improvement of throughput, it is of great importance to limit the use of solvent. With this purpose, a series of experiments varying $\alpha-P O$ concentration in the reaction medium were carried out. As it could be expected a maximum selectivity of $89 \%$ was achieved with lower $\alpha-P O$ concentration (Table 6 ). Additionally, the results of Table 7

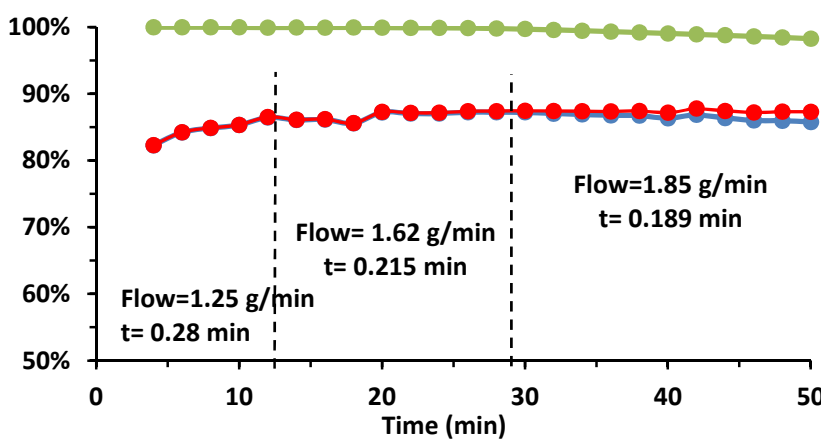

Figure 7. Effect of contact time over $\alpha$-PO conversion and CA yield and selectivity in fixed bed. Reaction conditions: $450 \mathrm{mg}$ of catalyst $(0.2<$ particle size $<0.4 \mathrm{~mm}), 150$ ㅇ $\mathrm{C}, 2 \mathrm{mmol}$ of $\alpha$-PO per $380 \mathrm{mg}$ of acetonitrile, different contact time from $0.28 \mathrm{~min}$ to $0.189 \mathrm{~min}$, i.e. total flow from 4.05 to $6 \mathrm{~mL} / \mathrm{min}$. $\bullet \mathrm{CA}$ yield, $\bullet \mathrm{CA}$ selectivity, $\bullet \alpha$-PO conversion. 
showed that a decrease of catalyst amount did not practically affect the CA selectivity and adjusting reaction time it was possible to produce CA with high yield and selectivity.

We believe that the ability of the post-synthesised Ti-Beta-PS-HT$\mathrm{Na}(0.1 \%)$ sample from readily available Al-Beta, whose catalytic performance for the isomerization of $\alpha-P O$ was improved thanks to the Na-exchange was shown. The excellent catalytic performance of Ti-Beta-PS-HT-Na(0.1\%) was due to the presence of tetrahedrally coordinated Ti sites in the silica-Beta framework whose Lewis acidity was matched through controlled hydrothermal incorporation of alkali-metal, with no Brønsted acidity. A proposed-mechanism of $\alpha$ $\mathrm{PO}$ isomerization by Lewis acid was reported some time ago (Scheme 2) [2].

We think that in the developed reaction conditions, $\alpha-P O$ concentration and catalyst amount, present unprecedentedly catalytic results in terms of CA production and selectivity. Likewise, in order to take a step forward in the development of a process and a catalyst validated at laboratory scale on an industrial scale, all these results were transferred to a study in flow. Moreover, the implementation of continuous process will contribute to sustainability and greenness.

\section{Reaction in fixed bed}

Since parameters of reaction are key factors to optimize process performance space velocity, temperature and solvent were optimized in fixed bed. The first experiments performed at $150^{\circ} \mathrm{C}$ with different space velocity from 1.25 to $1.85 \mathrm{~g} / \mathrm{min}$ per $0.45 \mathrm{~g}$ of TiBeta-PS-HT-Na. The data of conversion, yield and selectivity plotted in Figure 7 reflected that high CA yield and selectivity can be achieved up to $87 \%$ at complete conversion. Figure 8 illustrates the effect of temperature over $\alpha$-PO conversion and CA selectivity. The results proved that adsorption was affected strongly by temperature, since at 130 ㄷ $\mathrm{C}$ a constant decrease of conversion was observed from 98 to

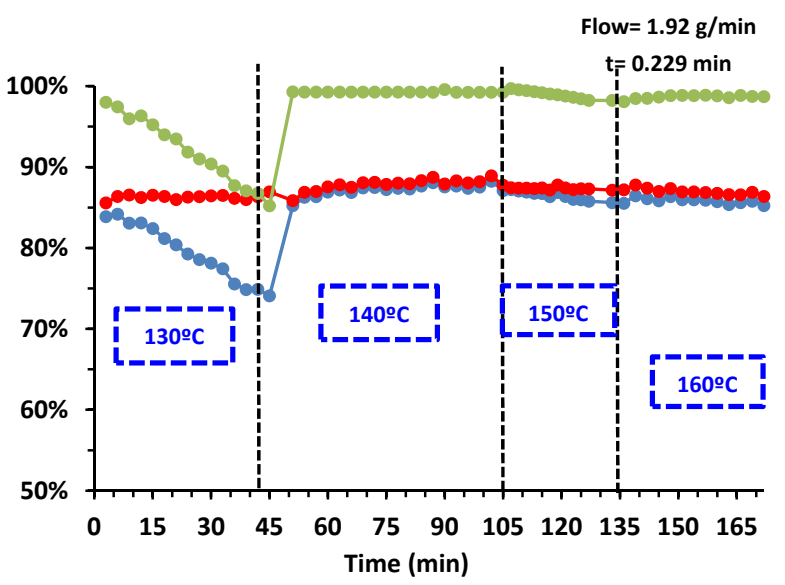

Figure 8. Effect of temperature over $\alpha$-PO conversion and CA yield and selectivity in fixed bed. Reaction conditions: $450 \mathrm{mg}$ of catalyst $(0.2<$ particle size $<0.4 \mathrm{~mm}), 2 \mathrm{mmol}$ of $\alpha$-PO per $380 \mathrm{mg}$ of acetonitrile, contact time from $0.229 \mathrm{~min}$, i.e. total flow from $5.28 \mathrm{~mL} / \mathrm{min}$. $\bullet$ CA yield, $\bullet$ CA selectivity, $\bullet \alpha$-PO conversion.

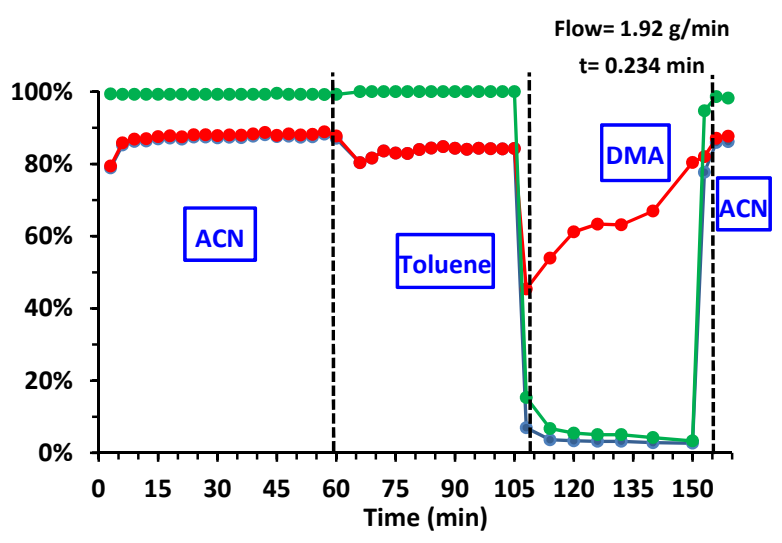

Figure 9. Effect of solvent over $\alpha-P O$ conversion and $C A$ yield and selectivity in fixed bed. Reaction conditions: $450 \mathrm{mg}$ of catalyst $(0.2<$ particle size $<0.4 \mathrm{~mm}), 2 \mathrm{mmol}$ of $\alpha-P O$ per $0.48 \mathrm{~mL}$ of solvent, contact time from $0.229 \mathrm{~min}$, i.e. total flow from 5.28 $\mathrm{mL} / \mathrm{min}$. $\bullet \mathrm{CA}$ yield, $\bullet \mathrm{CA}$ selectivity, $\bullet \alpha$-PO conversion.

$86 \%$ and an increase of temperature until to $140 \stackrel{\circ}{\circ}$ allowed recovering maximum conversion of $99 \%$ with CA selectivity close to $88 \%$. This result showed that deactivation of the catalyst by adsorption of reagent and product was a reversible process and possible by a slight increase of temperature. To continue the temperature was increased until $150^{\circ} \mathrm{C}$ and $160^{\circ} \mathrm{C}$, and really no drastic change over process selectivity was observed being around $87-86 \%$. Since temperature has a slight effect over process selectivity, $140{ }^{\circ} \mathrm{C}$ was determined as more adequate temperature. As we proceeded in batch reactor, the isomerization was set up using different solvents, feeding successively $\alpha$-PO solution in ACN, toluene, DMA and newly CAN (Figure 9). The results showed as in batch reactor that in the presence of DMA, the catalyst suffered a strong deactivation by adsorption of the solvent over the catalyst surface and metal active sites. Moreover, it was confirmed that is a

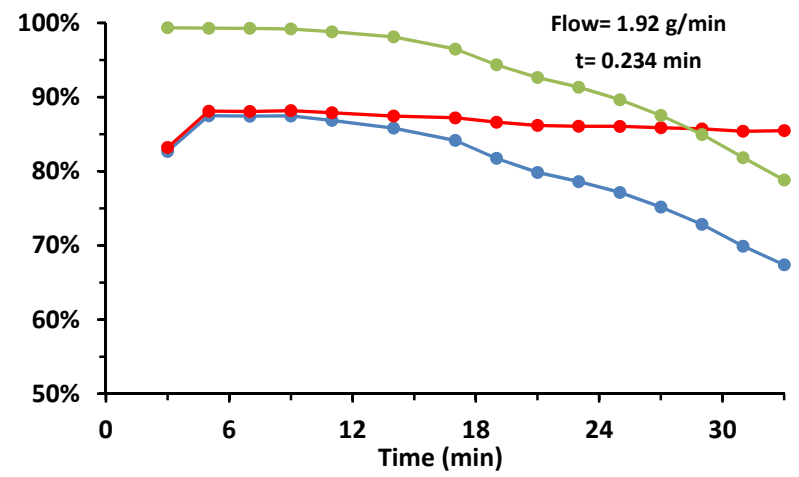

Figure 10. Catalytic performance of Ti-Beta-F for $\alpha-P O$ conversion and CA yield and selectivity in fixed bed. Reaction conditions: Fixed bed, $450 \mathrm{mg}$ of catalyst $(0.2<$ particle size $<0.4 \mathrm{~mm}$ ), $2 \mathrm{mmol}$ of $\alpha$-PO per $0.48 \mathrm{~mL}$ of solvent, contact time from $0.229 \mathrm{~min}$, i.e. total flow from $5.28 \mathrm{~mL} / \mathrm{min}$. $\bullet \mathrm{CA}$ yield, $\bullet$ CA selectivity, $\bullet \alpha-P O$ conversion. 


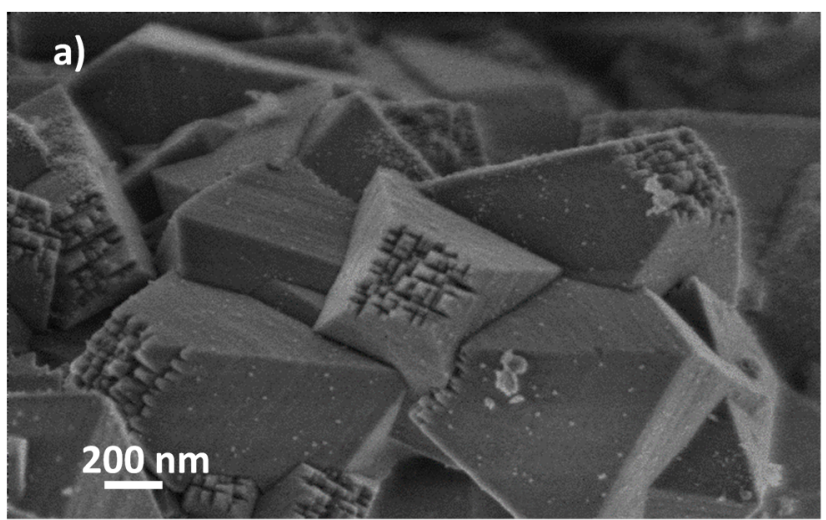

Figure 11. HRFESEM images of Ti-Beta-F and Ti-Beta-PS.

reversible process, since when new ACN solution of $\alpha$-PO was fed, the catalyst recovered its catalytic properties. The results in fixed bed were in agreement with those obtained in sealed vial under microwave. Moreover the excellent catalytic behaviour of the post synthetic material was clear as well as its stability. Indeed, when the study of temperature was performed $673 \mathrm{~g}$ of $\alpha$-PO were reacted over $0.45 \mathrm{~g}$ without observing catalyst deactivation. These results allowed a CA production of $225 \mathrm{~g} / \mathrm{g}_{\text {cat }} / \mathrm{h}$, using $\alpha$-PO in acetonitrile solution of $2.39 \mathrm{~mol} / \mathrm{L}$ and achieving $88 \%$ CA selectivity at maximum $\alpha$-PO conversion. These results in terms of selectivity are comparable to the best published results in the presence of $\mathrm{ZnBr}_{2}$ [4] or MOF [34] and slightly lower than in the presence of Ti-MCM22 [3]. Nevertheless, taking into account CA production, amount of catalyst, $\alpha-P O$ concentration and readily availability of the catalysts and cost, results reported in this work offer new opportunities to the development of greener industrial applications. Moreover if the catalytic data are compared to ones reported in 1998 by van Bekkum and co-workers [2] in flow using Ti-beta samples, the CA production is 490 times higher.

Finally the catalytic behaviour of conventional Ti-Beta sample prepared in fluoride media (Ti-Beta-F) in fixed bed was studied (Figure 10). A rapid deactivation of the Ti-Beta- $F$ was observed after 14 min of time on stream with a constant decay of $\alpha$-PO conversion with time. The catalytic behaviour of Ti-Beta- $F$ was very different to Ti-Beta-PS-HT-Na(0.1\%) plotted in Figure 8 and Figure 9 where the catalyst exhibited high catalytic performance and stability. Moreover, Ti-Beta-PS-HT-Na(0.1\%) presented ability to recover its catalytic properties after deactivation due to the use of inadequate temperature or solvent. This result was attributed to the morphology and size of the Ti-Beta-F particle. Indeed, the synthesis in fluoride media was characterised by long time of synthesis and the production of particles with large diameter. While the Ti-Beta-PS zeolite sample issued of two synthesis steps, the first of dealumination in acid media of Al-Beta and the second of ball-milling to incorporate $\mathrm{Ti}$, was expected to have smaller particle size due to acid treatment and friction effects. The difference in particle sizes was confirmed through high resolution field emission scanning electron microscopy. Images of Figure 11 illustrate the differences in the particles sizes of both samples close to 500 and $15 \mathrm{~nm}$ for Ti-Beta$\mathrm{F}$ and Ti-Beta-PS, respectively.

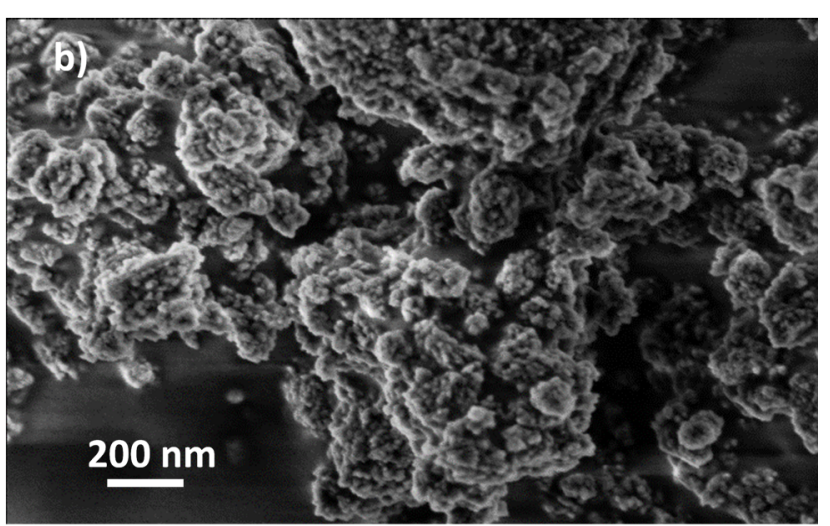

\section{Conclusions}

Post-synthesis Ti-Beta zeolite from readily available Al-Beta was prepared via ball-milling. The catalytic performance of the titanosilicate materials was successfully matched to maximize the CA selectivity through controlled hydrothermal $\mathrm{Na}$ and $\mathrm{Li}$ cation exchange and calcination step. The different population of open and closed Lewis active sites and weak Brønsted acid sites were characterized by IR of $\mathrm{CD}_{3} \mathrm{CN}$ adsorption. It was shown that the presence of alkali metal allowed not only to passivate the weak Brønsted acidity of $\mathrm{SiO}-\mathrm{H}$ adjacent to Ti-atoms but also to induce a weakening in the Ti-Lewis acidity strength. Then, the postsynthesised modified samples exhibited excellent catalytic performance for the $\alpha$-PO isomerization. Moreover, the optimization of the parameters of reaction led to develop a greener and sustainable process in both batch and flow reactor. From different point of views such as the preparation of readily, highly active, selective and stable catalyst, throughput, sustainability and cost, herein we considered that the selective solid catalysed $\alpha$-PO isomerization was reported with excellent results and opportunities.

\section{Conflicts of interest}

There are no conflicts to declare.

\section{Acknowledgements}

The authors are grateful for financial support from the Spanish Government by MAT2017-82288-C2-1-P and Severo Ochoa Excellence Program SEV-2016-0683. Mr. Pablo Ramos contribution to the experimental work is also gratefully acknowledged.

\section{Notes and references}

1 M. Stekrova, N. Kumar, A. Aho, I. Sinev, W. Grünert, J. Dahl, Jorma Roine, S. S. Arzumanov, P. Mäki-Arvela, D. Yu. M., Appl. Catal. A: General, 2014, 470, 162.

2 P. J. Kunkeler, J. C. van der Waal, J. Bremmer, B. J. Zuurdeeg, R. Downing and H. van Bekkum, Catal. Lett., 1998, 53, 135. 
3 M. Pitínová-Štekrová, P. Eliášová, T. Weissenberger, M. Shamzhy, Z. Musilová and J. Čejka, Catal. Sci. Technol., 2018, 8, 4690.

4 B. Arbushow, Chem. Ber., 1935, 68, 1430.

5 K. Arata and K. Tanabe, Chem. Lett., 1979, 8, 1017.

6 J. Kaminska, M. A. Schwegler, A. J. Hoefnagel and H. van Bekkum, Recl. Trav. Chim. Pays-Bas, 1992, 111, 432.

7 D. R. C. Huybrechts, L. De Bruycker and P. A. Jacobs, Nature, 1990, 345, 240.

8 C. Ferrini and H. W. Kouwenhoven, New Developments in Selective Oxidation, ed. G. Centi and F. Trifiro, Elsevier, Amsterdam, 1990, 53.

9 M. A. Camblor, A. Corma and J. Perez-Pariente, SP Pat. 9101 798, 1991.

10 M. A. Camblor, M. Costantini, A. Corma, L. Gilbert, P. Esteve, A. Martinez and S. Valencia, Chem. Commun., 1996, 11, 1339.

11 T. Blasco, M. A. Camblor, A. Corma, P. Esteve, A. Martinez, C. Prieto and S. Valencia, Chem. Commun., 1996, 20, 2367.

12 P. Li, G. Liu, H. Wu, Y. Liu, J. Jiang, P. Wu, J. Phys. Chem. C, 2011, 115, 3663.

13 J. Dijkmans, D. Gabriels, M. Dusselier, F. De Clippel, P. Vanelderen, K. Houthoofd, A. Malfliet, Y. Pontikes, B. F. Sels, Green Chem., 2013, 15, 2777.

14 C. Hammond, S. Conrad, I. Hermans, Angew. Chem., Int. Ed., 2012, 51, 11736.

15 P. Wolf, C. Hammond, S. Conrad and I. Hermans, Dalton Trans., 2014, 43, 4514.

16 C. Hammond, S. Conrad, I. Hermans, Angew. Chem. Int. Edit., 2012, 51, 11736.

17 J. Dijkmans, D. Gabriels, M. Dusselier, F. de Clippel, P. Vanelderen, K. Houthoofd, A. Malfliet, Y. Pontikes, B.F. Sels, Green Chem., 2013, 15, 2777.

18 S. Tolborg, I. Sadaba, C. M. Osmundsen, P. Fristrup, M. S. Holm, and T. Esben, ChemSusChem, 2015, 8, 613.

19 M. A Camblor, A. Corma, J. Perez-Pariente, Zeolites, 1993, 13, 82. 20 N. Garcia Vargas, S. Stevenson, D. F. Shantz, Microporous Mesoporous Mater., 2012, 152, 37.

21 T. Tatsumi, K. A. Koyano, Y. Shimizu, Appl. Catal. A, 2000, 200, 125.

22 C. B. Khouw, M. E. Davis, J. Catal., 1995, 151, 77.

23 Y. Kuwahara, K. Nishizawa, T. Nakajima, T. Kamegawa, K. Mori, H. Yamashita, J. Am. Chem. Soc., 2011, 133, 12462.

24 E. Taarning, S. Saravanamurugan, H. M. Spangsberg, J. Xiong, R. M. West, and C. H. Christensen, ChemSusChem, 2009, 2, 625.

25 R. Bermejo-Deval, M. Orazov, R. Gounder, S. j. Hwang, and M. E. Davis, ACS Catal., 2014, 4, 2288.

26 T. Blasco, M. A. Camblor, A. Corma, P. Esteve, J. M. Guil, A. Martınez, J. A. Perdigon-Melon, and S. Valencia, J. Phys. Chem. B, 1998, 102, 75.

27 R. K. Iler, The Chemistry of Silica; Wiley: New York, 1979.

28 M. J. Cordon, J. W. Harris, J. C. Vega-Vila, J. S. Bates, S. Kaur, M. Gupta, M. E. Witzke, E. C. Wegener, J. T. Miller, D. W. Flaherty, D. D. Hibbitts, and R. Gounder, J. Am. Chem. Soc., 2018, 140, 14244.

29 M. Boronat, P. Concepción, A. Corma, M. Renz, S. Valencia, J. Catal., 2005, 234, 111.

30 D. Gleeson, G. Sankar, C.R.A. Catlow, J.M. Thomas, G. Spano, S. Bordiga, A. Zecchina, C. Lamberti, Phys. Chem. Chem. Phys., 2000, 2, 4812.

31 R. Otomo, R. Kosugi, Y. Kamiya, T. Tatsumi and T. Yokoi, Catal. Sci. Technol., 2016, 6, 2787.

32 S. Imamura, T. Nakai, H. Kanai and T. Ito, J. Chem. Soc., Faraday Trans., 1995, 91, 1261.

33 G. Yang, L. Zhou, Scientific Reports, 2017, 7, 16113.
34 L. Alaerts, E. Séguin, H. Poelman, F. Thibault-Starzyk, P. A. Jacobs and D. E. De Vos, Chem. Eur. J., 2006, 12, 7353. 